\title{
COST ANALYSIS OF EARLY REHABILITATION AFTER STROKE IN COMPREHENSIVE CEREBROVASCULAR CENTRES IN THE CZECH REPUBLIC
}

\author{
Yvona Angerová1, Pavel Maršálek², Irina Chmelová3,4, Tereza Gueye ${ }^{5}$, Miroslav Barták ${ }^{6}$, Štěpán Uherek, \\ Jan Bř́za ${ }^{8}$, Vladimír Rogalewicz ${ }^{1}$ \\ ${ }^{1}$ Department of Rehabilitation Medicine, First Faculty of Medicine, Charles University and General University Hospital, Prague, Czech Republic \\ ${ }^{2}$ Rehabilitation Department, Masaryk Hospital, Ústí nad Labem, Czech Republic \\ ${ }^{3}$ Clinic of Rehabilitation and Physical Medicine, University Hospital Ostrava, Ostrava, Czech Republic \\ ${ }^{4}$ Department of Rehabilitation, Faculty of Medicine, University of Ostrava, Ostrava, Czech Republic \\ ${ }^{5}$ Stroke Unit Rehabilitation Department, First Faculty of Medicine, Charles University and General University Hospital, Prague, Czech Republic \\ ${ }^{6}$ Department of Addictology, First Faculty of Medicine, Charles University and General University Hospital, Prague, Czech Republic \\ ${ }^{7}$ Department of Biomedical Technology, Faculty of Biomedical Engineering, Czech Technical University in Prague, Kladno, Czech Republic \\ ${ }^{8}$ First Department of Surgery, First Faculty of Medicine, Charles University and General University Hospital, Prague, Czech Republic
}

\section{SUMMARY}

Objectives: The paper analyses real-world data on cost of treatment in patients after stroke hospitalized in early rehabilitation units within comprehensive stroke centres in the Czech Republic. This is the first study of the kind in the Czech Republic, while such information is extremely rare worldwide. Stroke treatment witnessed a dramatic development in the last years, when the main progress was due to establishment of specialized (comprehensive) stroke units incorporating also early rehabilitation. There is a general agreement among clinicians that early rehabilitation is beneficial for patients after stroke.

Methods: Costs of early rehabilitation after stroke were calculated by the micro-costing method alongside a pragmatic study in three Czech hospitals. Patients were transferred to specialized early rehabilitation units usually on 7 th to 14 th day after stroke onset and received four hours of interprofessional rehabilitation per day.

Results: The analysis of data collected during the prospective observational research of 87 patients proved significant differences between patients. The average costs of hospitalization were determined to be CZK 5,104 (EUR 194) per one day of intensive rehabilitation in seriously affected patients early after stroke. These costs differed significantly between hospitals ( $p$-value $<0.001$ ); the structure of direct costs was quite stable, though. About $60 \%$ of these costs were due to nursing and overhead, while no more than $15 \%$ were consumed by therapists.

Conclusions: The treatment of patients after stroke in specialized stroke units proved to be beneficial for the patients increasing the number of those re-integrated in family and community life.

Key words: stroke, early rehabilitation, comprehensive stroke centre, costs, real-world data

Address for correspondence: V. Rogalewicz, Department of Rehabilitation Medicine, First Faculty of Medicine, Charles University and General University Hospital, Albertov 7, 12808 Praha 2, Czech Republic. E-mail: vladimir.rogalewicz@lf1.cuni.cz

https://doi.org/10.21101/cejph.a6111

\section{INTRODUCTION}

Stroke is the second leading cause of death around the world $(1,2)$ and the cause of long-term disability. In the Czech Republic, there were 39,937 patients hospitalized with stroke, while 8,020 of them died in 2010. Per 100,000 patients, these figures are 379.17 and 76.14 , respectively (3).

In the United States, the costs of treatment and social security of patients after stroke have exceeded 43 billion US dollars annually. The General Health Insurance Company covering about $60 \%$ of the Czech population informed that they spent two billion Czech crowns (about EUR 74 million) for stroke treatment in 2016 (i.e., about $0.7 \%$ of the total Czech public health expenditures). Further finances are needed for social benefit payments for people after stroke and for informal care. Just the burden of informal caregiving for stroke survivors is USD 2.9 billion according to a recent US study (4). The total cost of acute stroke in England, Wales and Northern Ireland was reported to be GBP 3.6 billion taking into account the first five years after admission, while the mean costs per patient were GBP 46,039. There was a fivefold variation in the magnitude of costs between patients, ranging from GBP 19,101 to GBP 107,336 (5). Snozzi et al. showed the total cost of initial inpatient treatment and rehabilitation to be EUR 40,090 per hospitalized patient when estimated for an average 1-year observation period (6). Thus, stroke consumes approximately $2.9 \%$ of all inpatient costs in Switzerland.

Stroke treatment witnessed a dramatic development in the last years. Next to very new neurosurgical and neurological tech- 
nologies applied, the main progress was due to establishment of specialized (comprehensive) stroke units incorporating also early rehabilitation (7-9). In the Czech Republic, such stroke units were specified by the Ministry of Health in 2010 (10), established in 2012, and revised in 2015 (11). According to an Australian observational study (12), the establishment of a stroke unit not only improves treatment outcomes but also shortens the length of stay. Although the average cost of a patient treated in their stroke unit was approximately twice as high as in a conventional medical ward, the reduction of the length of stay and better post-hospitalization results led to the result that stroke units are cost-effective.

There is a general agreement among clinicians that early rehabilitation is beneficial for patients after stroke $(7,9,12-14)$. Next to clinical evidence, this argument is based on results of theoretical papers and animal experiments indicating that a narrow window of opportunity for brain plasticity and repair may exist, and the optimum period for change could be early after stroke $(15,16)$. The high-intensity rehabilitation therapy within the first 90 days is reported to be associated with a lower mortality risk than the low-intensity therapy among patients with mild to moderate stroke severity (17). The recent survey of Enderby et al. added new views to the discussion when characterizing a series of factors that might affect individual patient recovery. The necessity of personalized rehabilitation care for patients after stroke is accented (18).

The interpretation of the term "early rehabilitation" differs. The 2008 European stroke treatment guidelines only understood under early rehabilitation twenty or even thirty days after stroke, although running trials and discussions about much earlier interventions were mentioned $(19,20)$. Already six years later, the most common idea about early rehabilitation was seven days after stroke (21), and the AVERT trial was investigating a "very early mobilization" within 24 hours of stroke onset $(13,14)$. Up to now, next to the multicentre international AVERT trial with 2,104 patients, there were only rare small studies on the topic (21-23). Moreover, opposite promising results of pilot studies, the AVERT trial showed no significant difference between the mobilization within 24 hours of stroke and the standard care (which was, however, mostly within 48 hours). The problem about the best timing of early rehabilitation has stayed unambiguous. The recent American guidelines (7) recommend early, but not very early rehabilitation. Most authors speak about 2-7 days from stroke onset and stress that further research is necessary to study the optimal timing, frequency, and duration of early rehabilitation that probably has to be personalized for each patient $(13,18$, $22,23)$. Furthermore, not all patients reach the stroke unit or a hospital shortly after the stroke onset; the delay is a real problem, although outside the scope of this paper. Nelson et al. created an expert group investigating patients with stroke stressing that they often have several other co-occurring conditions and psychosocial issues; a stroke is said to occur in isolation (with no comorbid conditions) in less than $6 \%$ of patients. The co-occurring concerns may complicate stroke treatment and recovery (24). However, also these concerns stay outside the scope of our study.

There are 13 comprehensive cerebrovascular centres (consisting of neurosurgical, radiological, neurological and early rehabilitation units) and 32 stroke centres (consisting of neurological and early rehabilitation units) in the Czech Republic. As soon as possible, mostly between 7th and 14th day after stroke onset, patients are transferred to the early rehabilitation units, where they get three to four hours of interprofessional rehabilitation per day. At present, this care is not properly reimbursed by health insurance companies due to its intensity and the interprofessional team (including physicians, physiotherapists, occupational therapists, speech therapists, psychologists, nutrition therapists, and social workers). The first experience with rather intensive early rehabilitation is extraordinarily positive in the Czech hospitals.

In the General University Hospital (Czech acronym VFN) in Prague, 172 patients with stroke were admitted and provided with early rehabilitation in 2017. The average length of hospitalization was 30 days. Out of that, $63 \%$ of patients were discharged to their homes. They were provided with rehabilitation aids and educated how to use them, their apartments were evaluated on site, and changes for barrier-free homes were accomplished. Moreover, already in 1999, a rehabilitation day-care centre was established in the General University Hospital. Patients live at home and are transported to the centre every day, where they have an individually prepared interprofessional rehabilitation programme from 9 a.m. to 4 p.m. for 4 to 6 weeks. After a patient has been discharged from the stroke unit home, he/she can continue in the rehabilitation programme in this day-care centre. This is the reason why we can discharge a higher percentage of patients to community rehabilitation programmes.

There has been no comprehensive information about economic aspects of this care so far. The presented national multicentre pragmatic study was designed to determine costs of early rehabilitation after stroke during hospitalization of patients in Czech stroke units. The goal of this study is to calculate the hospitalization costs in patients after stroke at stroke units during the early rehabilitation therapy. We consider early rehabilitation to be a key tool for patient recovery after stroke, having also significant effect for their improvement in selfcare and common everyday activities (25). Next to the correct evidence-based care, the setting of the reimbursement system and the study of economic impact of stroke and stroke patient treatment are also essential.

\section{MATERIALS AND METHODS}

Real-world data of 94 patients were collected between 15 April and 27 October 2017 from three comprehensive cerebrovascular centres: General University Hospital in Prague - 30 patients; Masaryk Hospital in Ústí nad Labem - 34 patients; and University Hospital Ostrava - 30 patients. The study was approved by the Ethics Committee of the General University Hospital in Prague on 15 December 2016. The criteria for patient inclusion were stroke diagnosed within last two years (both ischaemic or haemorrhagic, primary or secondary as a complication of other type of healthcare, e.g., surgery or catheterisation). Transitory cases were not included. Patients with hospitalization shorter than four days were excluded regardless of the reason. The upper limit for the hospitalization length was set to 90 days; monitoring of a patient was finished after this limit had been reached, and the reason was recorded in the patient documentation. Each patient was included only once with the exception of an interruption of early rehabilitation due to complications treated in the same hospital. Subsequently, seven patients were excluded from the analyses, because the rehabilitation started more than three months after stroke in their case. The final number of patients included in the study was 87 . 
The study was pragmatic, so that each hospital followed their own standard procedures. Cost data or source data for cost calculations were collected by the bottom-up (micro-costing) method alongside the standard patient care. The staff recorded all data continuously in ten working forms (Table 1). Pieces, volumes or time spent were recorded in the working forms. These working forms were transferred to economic forms (each working form was accompanied by an economic form) multiplying amounts by their unit prices. Disposable materials, multiple-use aids and drugs were calculated by their average purchase prices, in case of multiple-use aids divided by the average number of uses. Nursing and therapeutic performances were calculated as the time spent in minutes multiplied by the average minute salary of the respective profession (profession-specific average gross annual salary divided by the number of minutes that the person actually spends with patients). The average salary data were taken from the Institute of Health Information and Statistics (IHIS) annual report (26). Prices of laboratory items were taken from the hospital financial statements. Prices for complementary therapies were estimated using the hospital financial statements. Prices of utilization of medical devices were calculated from their purchase prices (via depreciations), average maintenance prices and prices of consumables for one application.

Next to working and economic forms, there were also eight clinical forms: the basic and social form was used for recording basic personal data of each patient. The physician's form contained

Table 1. List of forms for data recording and pre-processing

\begin{tabular}{|l|}
\hline Working and economic forms \\
\hline Material form \\
\hline Nursing form \\
\hline Physiotherapeutic and occupational-therapeutic form \\
\hline Therapeutic form \\
\hline Medical device form \\
\hline Drug form \\
\hline Laboratory form \\
\hline Complementary therapy form \\
\hline Form of performances \\
\hline Used multiple-use aids form \\
\hline Clinical forms \\
\hline Basic and social form \\
\hline Physician's form \\
\hline Complication form \\
\hline Form of functional tests executed by the nursing staff \\
\hline $\begin{array}{l}\text { Form of Barthel index + extended Barthel index and the functional } \\
\text { independence measure }\end{array}$ \\
\hline $\begin{array}{l}\text { WHO International Classification of Functioning, Disability and Health } \\
\text { (ICF) Core Sets for Acquired Brain Injury (ABI) }\end{array}$ \\
\hline Form of further functional tests \\
\hline Note form \\
\hline Hospital forms \\
\hline Medical device identification form \\
\hline Overhead cost form \\
\hline
\end{tabular}

dates (stroke onset, hospitalization, transfer to early rehabilitation unit, discharge from hospital) and physician's independent evaluation of the patient's functional abilities. The complication form was used for recording of any state different from normal course of the rehabilitation therapy. Further clinical forms served to record results of various functional tests carried out during the hospitalization (these data are not used in the present study). The note form served for any further notes concerning the patient and his/her treatment; it was managed by the physician, but anybody of the staff could use it. Finally, general information that is not related to individual patients was collected in two hospital forms. The medical device identification form was used to record costrelated data about all applied devices (a separate form for each particular device). These forms were filled out in collaboration with the methodologist of the project and contained rather usual than average data. The overhead cost form was filled out by the financial or controlling departments of the hospitals and contained best possible estimates of the overhead items.

At the end of the clinical part of the study, software was used to merge data into one excel table. The data were optically checked for possible errors, and suspicious fields were checked in the patient's hospital documentation. Statistical analyses were executed in the R statistical software. Next to basic statistical characteristics, most calculations consisted of calculating frequencies, sums of costs of individual interventions, and their weighted averaging and consolidations. One-way ANOVA was used to test the inter-hospital differences.

\section{RESULTS}

\section{Description of Patient Sample}

The age of 87 patients included in the study was in the range from 31 to 95 years with the average of 70.5 and median of 71 years. Table 2 shows age distribution of patients. The stroke had ischaemic-embolic aetiology in 21 cases, other ischaemic aetiology in 57 cases, haemorrhagic in 7 cases, and in 2 cases the origin was not recorded. The primary therapy was conservative in 50 patients, thrombolytic in 26 patients, intravascular in 9 patients, and surgical in 2 patients. The laterality in hemiparesis was balanced (left-sided in 41 patients, right-sided in 41 patients, and no or unrecorded in 5 patients). The patients were transferred to the rehabilitation unit 4-69 days after stroke with the average of 16.2 days and median of 11 days (Table 2). The average length of hospitalization was 22.2 days (median 20 days, min. 4 days, max. 59 days). However, due to the pragmatic character of the study, the length of hospitalization differed between the hospitals significantly $(p<0.000003)$ (Fig. 1).

\section{Cost Analysis}

The average costs per hospitalization of one patient differed significantly between hospitals, partly because different lengths of hospitalization. One-day averages seem to be more appropriate for further analyses. The one-day average values do not depend on the length of hospitalization $(r=0.11, p=0.296)$. Average hospitalization costs are shown in Table 3 . We tested the differences between hospitals by a one-way ANOVA $(F=49.15406$, 
Table 2. Patient age distribution and days between stroke onset and admission to rehabilitation unit $(N=87)$

\begin{tabular}{|c|c|c|c|c|c|}
\hline \multicolumn{3}{|c|}{ Patient age distribution } & \multicolumn{2}{c|}{$\begin{array}{c}\text { Days between stroke onset and } \\
\text { admission to rehabilitation unit }\end{array}$} \\
\hline & $\mathrm{n}$ & $\%$ & & $\mathrm{n}$ & $\%$ \\
\hline $0-39$ & 1 & 1.1 & $<7$ & 10 & 11.5 \\
\hline $40-49$ & 4 & 4.6 & $7-13$ & 40 & 46.0 \\
\hline $50-59$ & 14 & 16.1 & $14-20$ & 16 & 18.4 \\
\hline $60-69$ & 21 & 24.1 & $21-27$ & 6 & 6.9 \\
\hline $70-79$ & 23 & 26.4 & $28-34$ & 10 & 11.5 \\
\hline $80-89$ & 20 & 23.0 & $35-60$ & 3 & 3.4 \\
\hline $90+$ & 4 & 4.6 & $>60$ & 2 & 2.3 \\
\hline
\end{tabular}

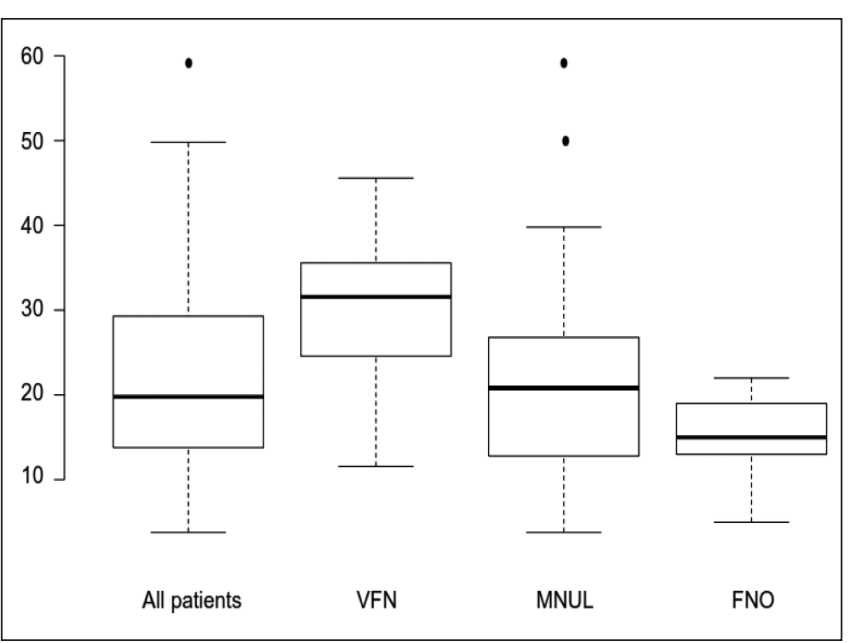

Fig. 1. Length of hospitalization in days.

VFN - General University Hospital; MNUL - Masaryk Hospital in Ústí nad Labem; FNO - University Hospital Ostrava

All data together and three involved hospitals individually

$\mathrm{p}<0.001)$. Thus, the differences of one-day hospitalization costs between hospitals were proved to be statistically significant. However, the structure of direct costs was quite stable. The left chart in Figure 2 shows the distribution of costs among particular types of direct costs ("other" combines laboratory examinations, consortia and complement treatments), while the right one shows the sub-distribution of the personal cost category.

\section{DISCUSSION}

The goal of the study was a calculation of the hospitalization costs in patients after stroke at stroke units during the early rehabilitation therapy. The wider objective was to inform healthcare insurance companies about the actual costs of this therapy as a basis for new reimbursement rates negotiation. The hypothesis behind the trial was that such intensive rehabilitation has not been adequately reimbursed yet. Early rehabilitation is a key tool for patient recovery after stroke, and it has a significant effect for the patient's improvement in selfcare and common everyday activities (25). Next to the appropriate evidence-based care, also setting of the reimbursement system and investigation of economic impact of stroke and stroke patient treatment are essential.

Results published in this field show a notable difference between the situation in the Czech Republic and abroad. This difference mirrors in the number of published papers and accomplished studies, but also in the level of the early interprofessional rehabilitation system for post-stroke care generally. While there are no clinical studies investigating either clinical effectiveness or cost-effectiveness of early rehabilitation after stroke, and the experiment underlying this paper was the first experiment of the kind in the Czech Republic, foreign trials studied the very early therapeutic intervention applied within 24 hours since the stroke onset in emergency neurological units (14, 27-29), i.e., the mobilization connected with the early conservative or surgical therapy after stroke. The issue of an early application of specialized therapy in neurology departments is characterized by a few hours (e.g., the possibility of thrombolysis), and it is comparable in the Czech Republic and other EU countries. Our study focused on the next phase of the therapeutic process starting with patient's transfer from the neurological ICU to the early rehabilitation unit within a comprehensive cerebrovascular/stroke centre (10). Hence, the time of our early interprofessional rehabilitation is counted from the transfer of the patient to the early rehabilitation unit. In the authors' opinion, this approach is an important success and a step towards undoubtedly more effective treatment of patients after stroke aimed at patient's return to the best possible quality of life.

Costs of early rehabilitation after stroke were calculated by the micro-costing method alongside a pragmatic study in three Czech hospitals. The analysis of real-world data collected during the prospective observational research of 87 patients recovering in early rehabilitation units proved significant differences between patients within a single diagnosis, the stroke. The differences can be found in all studied aspects of the early rehabilitation of patients after stroke, i.e., in costs, clinical outcomes, as well as in cost effectiveness ratios. This paper focused on the cost features; a study of outcomes and utilities was published elsewhere including a detailed discussion (30).

The analysis showed differences in patient management, length of stay, as well as in the costs between the particular hospitals. Significant cost differences were caused predominantly by hospitalization length related also to the seriousness of condition in patients transferred from neurology departments. The differences

Table 3. Costs of hospitalization at rehabilitation department

\begin{tabular}{|l|c|c|c|c|}
\hline Average costs per patient & All together & VFN & MNUL & FNO \\
\hline \multirow{2}{*}{ Hospitalization } & CZK 114,489 & CZK 174,984 & CZK 87,754 & CZK 80,209 \\
\cline { 2 - 5 } & EUR 4,348 & EUR 6,646 & EUR 3,333 & EUR 3,046 \\
\hline \multirow{2}{*}{ One day average } & CZK 5,103.61 & CZK 6,029.52 & CZK 4,095.85 & CZK 5,266.18 \\
\cline { 2 - 5 } & EUR 193.83 & EUR 229.00 & EUR 155.56 & EUR 200.01 \\
\hline
\end{tabular}

Values in CZK are results of the cost analysis; values in EUR are converted by the 2017 Czech National Bank average exchange rate EUR $1=$ CZK 26.330

VFN - General University Hospital; MNUL - Masaryk Hospital in Ústí nad Labem; FNO - University Hospital Ostrava 


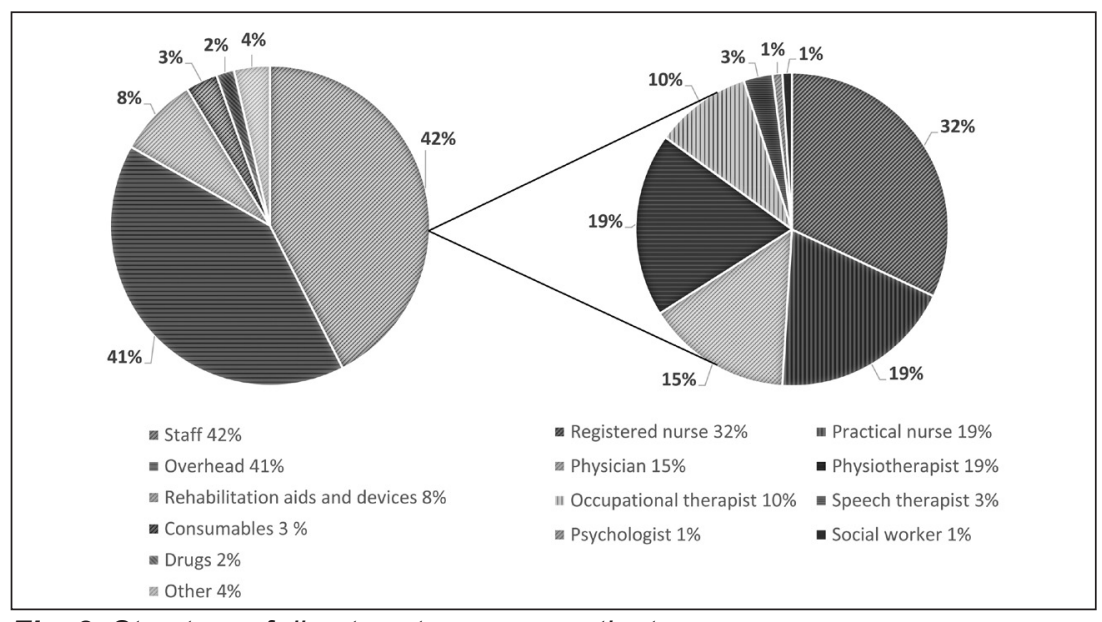

Fig. 2. Structure of direct costs per one patient.

Staff costs ( $42 \%$ in the first chart) are divided in the other chart to sub-categories.

in the average one-day costs were caused by particular hospital treatment procedures and managerial processes. However, all these differences appeared to be quite complex and non-trivial, and thus they were thoroughly analysed in a separate paper (31).

The authors expected that higher costs of early rehabilitation would be driven above all by the number of staff necessary and by the time intensity of the care. However, it appeared that the requirements on therapists mirror only slightly in the costs when personal costs on physiotherapists and occupational therapists make no more than $15 \%$ of the total costs. In comparison with that, the costs spent just to keep the patient in the hospital generate up to $63 \%$ (overhead costs $41 \%$ and nursing $22 \%$ ) of all costs.

There are only few published results concerning costs of rehabilitation in patients with stroke. Tay-Teo et al. (28) published costs recorded alongside the AVERT phase II trial that can be labelled as a pilot study (27). The single-blinded study was carried out in two large teaching hospitals in Melbourne, Australia, where 71 patients (38 in the very early mobilization arm, and 33 in the standard care arm) were recruited between May 2005 and February 2006. The costs spent for rehabilitation (inpatient, outpatient, and at home) between stroke onset and 3-month follow-up was AUD 14,390 (EUR 8,639) for the very early mobilization arm, and AUD 19,050 (EUR 11,437) for the standard care arm. Recently, first cost data collected alongside the AVERT phase III trial with 2,104 patients from 56 stroke units in Australia, New Zealand, Malaysia, Singapore, and UK were published (32). The results collected alongside a controlled trial (i.e., not real-world data) are affected by extremely different price levels in the covered countries. Nevertheless, the very early mobilization approach showed no significant differences in costs as compared with the usual care.

Lahiry et al. (33) recently published an economic analysis focused above all on pre-hospital phase in patients with suspected acute stroke in Australian rural areas. They recommended an improved protocol for the care for stroke patients outside large urban agglomerations and compared all costs under their protocol and in current standard care. In their cost analysis, rehabilitation was valued by AUD 6,950 (EUR 4,708) in the standard care arm, and AUD 7,775 (EUR 5,267) in their new protocol arm.

However, these figures are incomparable because there was variability in the length and intensity of the rehabilitation care, which is true also for our Czech results. In our experiment, the early rehabilitation provided at specialised stroke unit was valued at CZK 114,489 (EUR 4,348). It is also necessary to take into account the materially lower price level in the Czech Republic that manifests itself above all in personal costs.

Since the early rehabilitation has been introduced to stroke units, most patients are dismissed to the home care (in our multicentre experiment $52 \%$, in VFN even $63 \%$ ), while most patients were transferred to the chronic disease hospitals without any rehabilitation before. This fact has an important effect on expenses incurred to the healthcare budget. Subsequently it has a notable effect also to the social affairs budget decreasing the number of people applying for social benefits and disability pension beneficiaries. Future research should be focused on these issues on the border between the healthcare and social areas.

There are some limitations to the study that might affect the results. First, there is the limited time period (one year) and research capacity. The main limitations are the relatively small samples from the individual hospitals as well as the small sample of hospitals themselves. However, the sample variability allowed for an estimation of average costs of early rehabilitation in patients after stroke, which was the main goal of this study. Other limitations that might have affected the analysis of clinical outcomes and comparison of the involved stroke centres were included in the respective publications $(30,31)$.

\section{CONCLUSIONS}

The treatment of patients after stroke in specialized stroke units proved to be beneficial for the patients increasing the number of those re-integrated in family and community life. However, a greater focus on return-to-work as part of stroke rehabilitation may be of value for patients of working age. It is also possible to use the experience and assistance of the prevocational centres mentioned above. Early rehabilitation is an important part of the therapeutic process. We calculated the costs of the early inpatient rehabilitation in the setting of Czech stroke units. The average price was determined to be CZK 5,104 (EUR 194) per one day of inpatient intensive rehabilitation in seriously affected patients early after stroke. About $60 \%$ of these costs are due to nursing and overhead, while no more than $15 \%$ is the part consumed by therapists. 


\section{Acknowledgement}

The research leading to this paper was supported by the grant No. 410004194 provided by the General Health Insurance Company of the Czech Republic from their Secondary Prevention Fund.

The authors would like to express their gratitude to prof. Olga Švestková who passed away in December 2018. She was the conceptual originator of this research. She also started to work on publication of the results.

Her work was suddenly interrupted. We tried to continue in promoting the results thinking of her enormous enthusiasm for rehabilitation which inspired us.

\section{Conflict of Interests}

None declared

\section{REFERENCES}

1. Feigin VL, Forouzanfar MH, Krishnamurthi R, Mensah GA, Connor M, Bennett DA, et al. Global and regional burden of stroke during 19902010: findings from the Global Burden of Disease Study 2010. Lancet. 2014;383(9913):245-55.

2. Feigin VL, Roth GA, Naghavi M, Parmar P, Krishnamurthi R, Chugh S, et al. Global burden of stroke and risk factors in 188 countries, during 1990-2013: a systematic analysis for the Global Burden of Disease Study 2013. Lancet Neurol. 2016;15(9):913-24.

3. Institute of Health Information and Statistics of the Czech Republic. Cerebrovascular diseases - hospitalized patients and deaths in the Czech Republic in 2003-2010. Aktuální informace [Internet]. 2012 Feb [cited 2021 Apr 1];(3):1-27. Available from: https:/www.uzis.cz/sites/default/ files/knihovna/03 12.pdf.

4. Joo H, Wang GJ, Yee SL, Zhang P, Sleet D. Economic burden of informal caregiving associated with history of stroke and falls among older adults in the US. Am J Prev Med. 2017;53(6):S197-204.

5. Xu XM, Vestesson E, Paley L, Desikan A, Wonderling D, Hoffman A, et al. The economic burden of stroke care in England, Wales and Northern Ireland: using a national stroke register to estimate and report patient-level health economic outcomes in stroke. Eur Stroke J. 2018;3(1):82-91.

6. Snozzi P, Blank PR, Szucs TD. Stroke in Switzerland: social determinants of treatment access and cost of illness. J Stroke Cerebrovasc Dis. 2014;23(5):926-32.

7. Powers WJ, Rabinstein AA, Ackerson T, Adeoye OM, Bambakidis NC, Becker K, et al. 2018 Guidelines for the Early Management of Patients With Acute Ischemic Stroke: a guideline for healthcare professionals from the American Heart Association/American Stroke Association. Stroke. 2018;49(3):e46-110.

8. Škoda O, Herzig R, Mikulík R, Neumann J, Václavík D, Bar M, et al. Clinical Guideline for the Diagnostics and Treatment of Patients with Ischemic Stroke and Transitory Ischemic Attack - version 2016. Ces Slov Neurol Neurochir. 2016;79(3):351-63. (In Czech.)

9. Hamann GF, Müller R, Alber B, Widder B. Treatment in acute stroke stroke unit is mandatory. Neurol Psych Brain Res. 2016;22(2):105-9.

10. Ministry of Health of the Czech Republic. Care of patients with cerebrovascular disease in the Czech Republic. Věstník MZ ČR. 2010;2010(2):213. (In Czech.)

11. Ministry of Health of the Czech Republic. List of highly specialized centers for the care of patients after acute stroke. Věstník MZ ČR. 2015;2015(11):52-6. (In Czech.)

12. Zhai S, Gardiner F, Neeman T, Jones B, Gawarikar Y. The cost-effectiveness of a stroke unit in providing enhanced patient outcomes in an Australian teaching hospital. J Stroke Cerebrovasc Dis. 2017;26(10):2362-8.

13. Langhorne P, Wu O, Rodgers H, Ashburn A, Bernhardt J. A Very Early Rehabilitation Trial after stroke (AVERT): a Phase III, multicentre, randomised controlled trial. Health Technol Assess. 2017;21(54):1-120.

14. Bernhardt J, Langhorne P, Lindley RI, Thrift AG, Ellery F, Collier J, et al. Efficacy and safety of very early mobilisation within $24 \mathrm{~h}$ of stroke onset (AVERT): a randomised controlled trial. Lancet. 2015;386(9988):46-55.

15. Murphy TH, Corbett D. Plasticity during stroke recovery: from synapse to behaviour. Natur Rev Neurosci. 2009;10(12):861-72.
16. Krakauer JW, Carmichael ST, Corbett D, Wittenberg GF. Getting neurorehabilitation right: what can be learned from animal models? Neurorehabil Neural Repair. 2012;26(8):923-31.

17. Hsieh CY, Huang HC, Wu DP, Li CY, Chiu MJ, Sung SF. Effect of rehabilitation intensity on mortality risk after stroke. Arch Phys Med Rehabil. 2018;99(6):1042-8.

18. Enderby P, Pandyan A, Bowen A, Hearnden D, Ashburn A, Conroy P, et al. Accessing rehabilitation after stroke - a guessing game? Disabil Rehabil. 2017;39(7):709-13.

19. Hacke W, Ringleb PA, Bousser M-G, Ford G, Bath P, Brainin M, et al. Guidelines for management of ischaemic stroke and transient ischaemic attack 2008 - The European Stroke Organisation (ESO) Executive Committee and the ESO Writing Committee. Cerebrovasc Dis. 2008;25(5):457-507.

20. Quinn TJ, Paolucci S, Sunnerhagen KS, Sivenius J, Walker MF, Toni $\mathrm{D}$, et al. Evidence-based stroke rehabilitation: an expanded guidance document from the European Stroke Organisation (ESO) guidelines for management of ischaemic stroke and transient ischaemic attack 2008. J Rehabil Med. 2009;41(2):99-111.

21. Lynch E, Hillier S, Cadilhac D. When should physical rehabilitation commence after stroke: a systematic review. Int J Stroke. 2014;9(4):468-78.

22. Miranda Rocha AR, Martinez BP, Maldaner da Silva VZ, Forgiarini Junior LA. Early mobilization: Why, what for and how? Med Intensiva. 2017;41(7):429-36.

23. Li ZY, Zhang XM, Wang K, Wen J. Effects of early mobilization after acute stroke: a meta-analysis of randomized control trials. J Stroke Cerebrovasc Dis. 2018;27(5):1326-37.

24. Nelson ML, McKellar KA, Munce S, Kelloway L, Hans PK, Fortin $\mathrm{M}$, et al. Addressing the evidence gap in stroke rehabilitation for complex patients: a preliminary research agenda. Arch Phys Med Rehabil. 2018;99(6):1232-41.

25. Yagi M, Yasunaga H, Matsui H, Morita K, Fushimi K, Fujimoto M, et al. Impact of rehabilitation on outcomes in patients with ischemic stroke: a nationwide retrospective cohort study in Japan. Stroke. 2017;48(3):740-6.

26. Institute of Health Information and Statistics of the Czech Republic. Healtcare in the Czech Republic: personnel capacities and remuneration 2018 [Internet]. Prague: Institute of Health Information and Statistics of the Czech Republic; 2019 [cited 2021 Apr 1]. Available from: https:// www.uzis.cz/res/f/008281/nzis-rep-2019-e04-personalni-kapacityodmenovani-2018.pdf. (In Czech.)

27. Bernhardt J, Dewey H, Thrift A, Collier J, Donnan G. A very early rehabilitation trial for stroke (AVERT) phase II safety and feasibility. Stroke. 2008;39(2):390-6.

28. Tay-Teo K, Moodie M, Bernhardt J, Thrift AG, Collier J, Donnan G, et al. Economic Evaluation alongside a Phase II, Multi-Centre, Randomised Controlled Trial of Very Early Rehabilitation after Stroke (AVERT). Cerebrovasc Dis. 2008;26(5):475-81.

29. Sheppard L, Dewey H, Bernhardt J, Collier JM, Ellery F, Churilov L, et al. Economic Evaluation Plan (EEP) for A Very Early Rehabilitation Trial (AVERT): an international trial to compare the costs and costeffectiveness of commencing out of bed standing and walking training (very early mobilization) within $24 \mathrm{~h}$ of stroke onset with usual stroke unit care. Int J Stroke. 2016;11(4):492-4.

30. Angerova Y, Marsalek P, Chmelova I, Gueye T, Uherek S, Briza J, et al. Cost and cost-effectiveness of early inpatient rehabilitation after stroke varies with initial disability: the Czech Republic perspective. Int J Rehabil Res. 2020;43(4):376-82.

31. Kratochvílová A, Rogalewicz V, Angerová Y, Gueye T, Maršálek P, Chmelová I, et al. Early rehabilitation after stroke in comprehensive cerebrovascular centres in the Czech Republic: a comparison of three stroke units. Kontakt. Ahead of print. doi: 10.32725/kont.2021.013.

32. Gao L, Sheppard L, Wu O, Churilov L, Mohebbi M, Collier J, et al. Economic evaluation of a phase III international randomised controlled trial of very early mobilisation after stroke (AVERT). BMJ Open. 2019;9(5):e026230. doi: 10.1136/bmjopen-2018-026230.

33. Lahiry S, Levi C, Kim J, Cadilhac DA, Searles A. Economic evaluation of a pre-hospital protocol for patients with suspected acute stroke. Front Public Health. 2018;6:43. doi: 10.3389/fpubh.2018.00043. 